\title{
Synthesis and Size-Controllable Self-Assembly of A Novel Amphiphilic Hyperbranched Multi-Arm Copolyether
}

\author{
Yiyong Mai, Yongfeng Zhou*, Deyue Yan* \\ College of Chemistry and Chemical Engineering, Shanghai Jiao Tong \\ University, 800 Dongchuan Road, Shanghai 200240, P. R. China
}

Supporting Information

\section{S1. DSC measurement}

The DSC curves are shown in Figure S1 and the data of $T_{\mathrm{g}}$ are listed in Table 1 (in Main Text). Hyperbranched PEHO has a $T_{\mathrm{g}}$ about $40{ }^{\circ} \mathrm{C}^{1}$ and the linear PPO exhibits a $T_{\mathrm{g}}$ around $-75^{\circ} \mathrm{C}^{2}$. For PEHO-star-PPO samples, $T_{\mathrm{g}}$ gradually falls from $-21.4{ }^{\circ} \mathrm{C}$ to $-66.7^{\circ} \mathrm{C}$ with increasing $R_{\mathrm{A} / \mathrm{C}}$ from 0.8 to 9.2. The tendency is similar to that given by Frey et al. for the hyperbranched copolymers with PPO arms. ${ }^{2}$ As pointed out by Frey et al., the flexible PPO segments can be considered as the spacer units introduced between the hyperbranched scaffold and the hydroxyl end groups, leading to the decreases of the functional group density and the flexibility of the whole multi-arm molecule. Therefore, the flexibility of PEHO-star-PPO is between those of PEHO and PPO segments, and approaches the flexibility of linear PPO with increasing the amount of attached PPO arms. It is well-known that a copolymer will show only one $T_{\mathrm{g}}$ if the different segments of the copolymer are miscible. ${ }^{2,3}$ Taking account of the similar polyether components of PEHO cores and PPO arms, we consider that PEHO cores and PPO arms are probably miscible and may form a mixed phase. Therefore, only one $T_{\mathrm{g}}$ in the DSC curve for every PEHO-star-PPO copolymer is observed. 


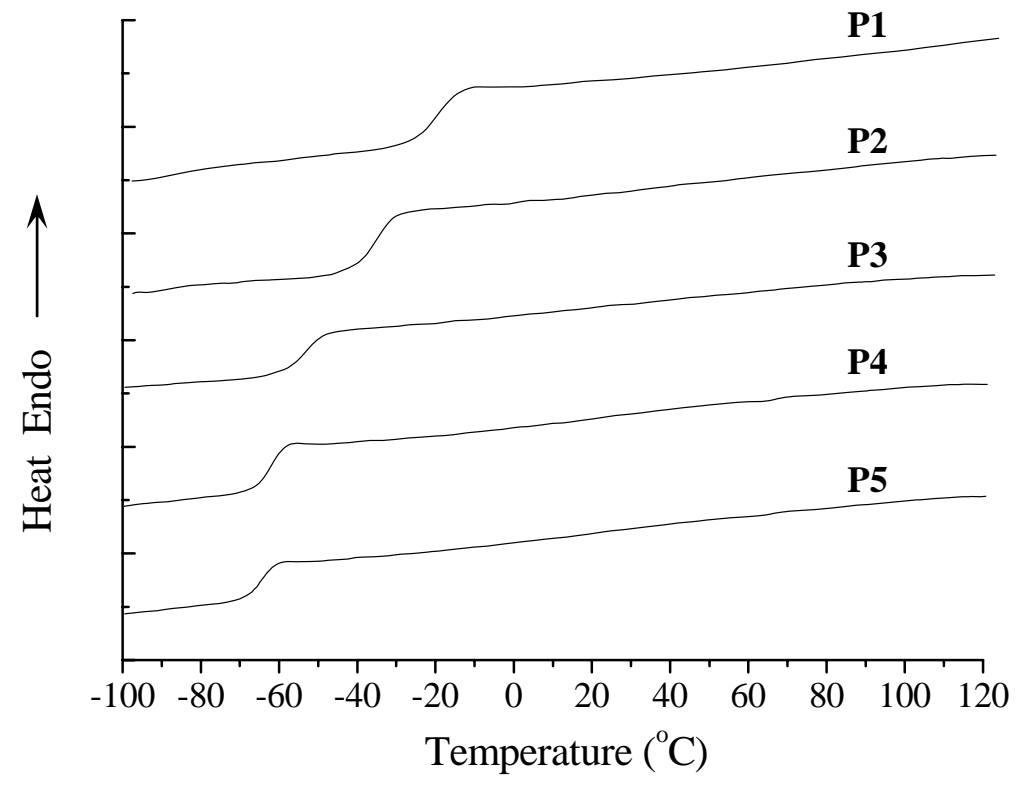

Figure S1. DSC curves of PEHO-star-PPO copolymers

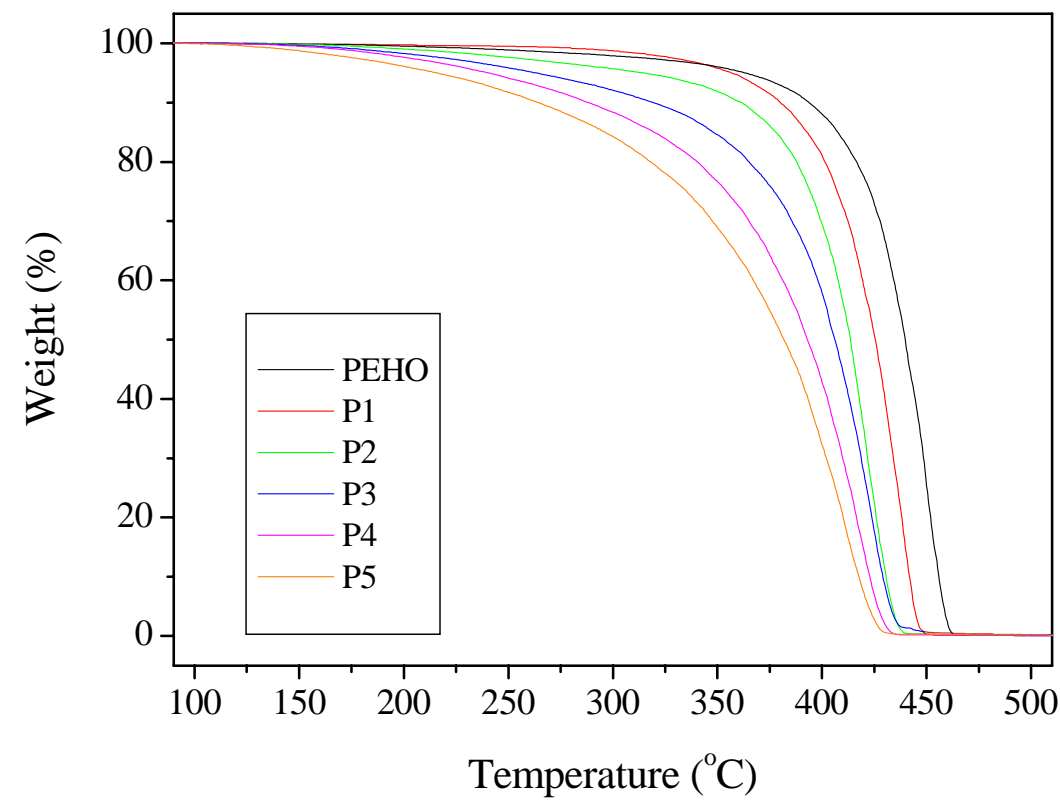

Figure S2. TGA plots of PEHO and PEHO-star-PPO copolymers

\section{S2. TGA analysis}

The TGA plots given in Figure S2 and the data listed in the last column of Table 1 (in Main Text) indicate that the decomposition temperature $\left(T_{\mathrm{d}}\right)$ also falls as $R_{\mathrm{A} / \mathrm{C}}$ increases. The only one $T_{\mathrm{d}}$ observed on the TGA plot for every copolymer sample is possibly attributed to the similar composition of the cores and arms of PEHO-star-PPO copolyethers. 


\section{S3. SEM observations}

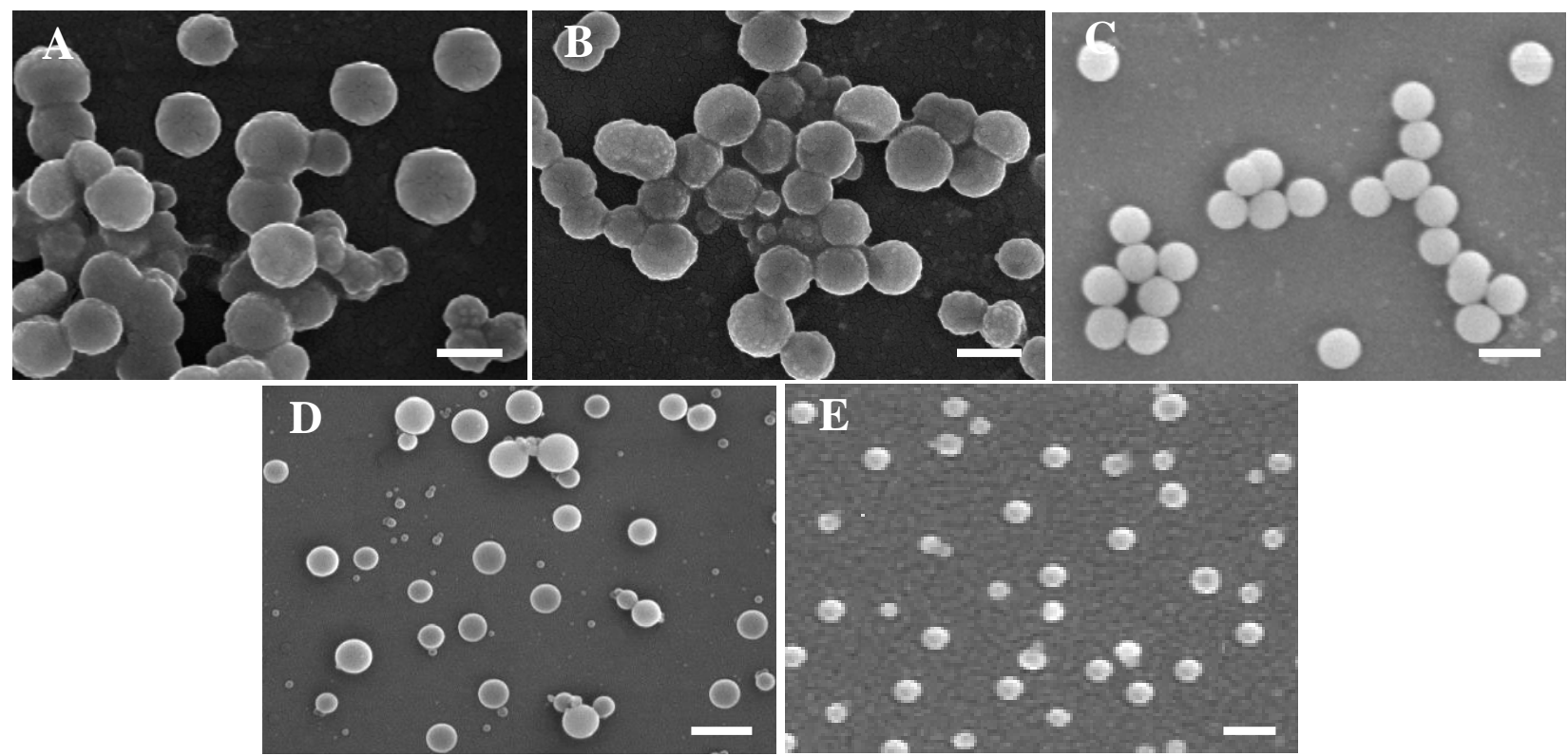

Figure S3. SEM photographs (A-E) of P1 5. A) P1 micelles, B) P2 micelles, C) P3 micelles, D) P4 micelles, E) P5 micelles. The scale bars stand for $250 \mathrm{~nm}$.

S4. The various phenomena of the self-assembly of original and end-capped PEHO-star-PPO samples

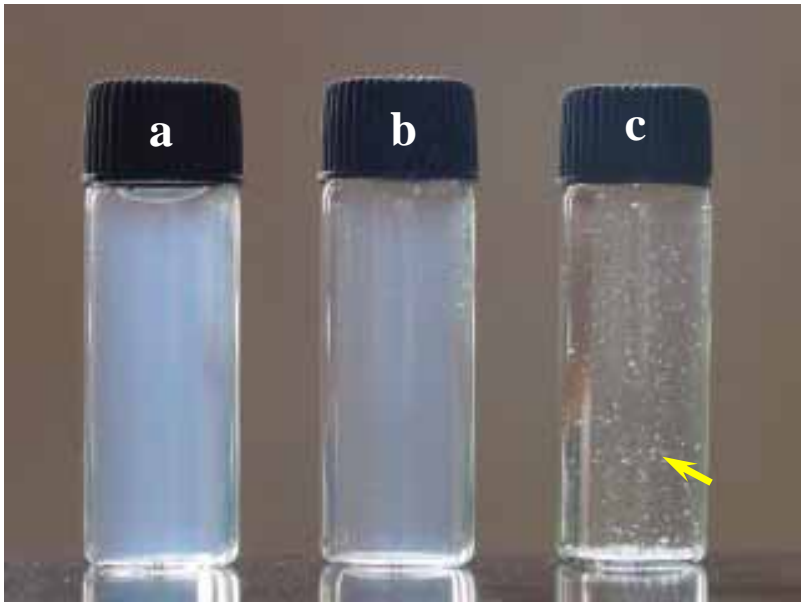

Figure S4. The different phenomena of the self-assembly of original and end-capped samples of $\mathrm{P} 2$. a: original sample; $\mathbf{b}$ : $f \approx 0.1$ sample; and $\mathbf{c}: f \approx 0.6$ sample. Some precipitate (yellow arrow) was found in $\mathbf{c}$. 


\section{References}

(1). Mai, Y.; Zhou, Y.; Yan, D. Chem. J. Chinese U. 2004, 25, 1373.

(2). Sunder, A.; Mülhaupt, R.; Frey, H. Macromolecules 2000, 33, 309.

(3). Schmalz, H.; Knoll, A.; Müller, A. J.; Abetz, V. Macromolecules 2002, 35, 10004. 